\title{
ON COMBINATORIAL ARRANGEMENTS
}

KULENDRA N. MAJUMDAR

An arrangement of objects of $v$ different varieties into $b$ blocks (or sets) such that (i) no two blocks are identical (i.e. contain the same varieties), (ii) a variety occurs at most once in a block, (iii) any pair of varieties occurs together in $\lambda$ blocks, $\lambda \neq 0$, (iv) every block contains $k$ varieties, $k<v$, is called a balanced incomplete block design. These designs are of great use in applied statistics.

From (i), (ii), (iii), and (iv) it easily follows [1] that (v) all the varieties occur in the whole design an equal number of times, $r$, say, where $r=\lambda(v-1) / k-1$. But it is not difficult to see by constructing examples that the conditions (i), (ii), (iii), and (v) in general do not imply (iv).

About four years ago, Ryser [2] proved an interesting result (given here in an essentially equivalent form) that for symmetrical designs (i.e. designs in which $b=v$ ) conditions (i), (ii), (iii), and (v) imply (iv). In this note we give an extension of this result. To this end we first prove a general result on matrices given in Theorem 1. By column sum we shall mean the sum of the elements in a column.

THEOREM 1. If two conformable matrices $A, B$ (whose elements may belong to any given field) are such that

(i) column sums of $C$, where $C=A B$, are equal, to $c$ say,

(ii) column sums of $B$ are equal to, say $b$, then any column sum of $A$ is $c / b$ provided the rank of $B$ is equal to the number of its rows.

Let $A$ and $B$ be $m \times r$ and $r \times n$ matrices respectively. Since rank $B=r, n \geqq r$. Denote by 1 the unit element of the field. Pre-multiply the relation $A B=C$ by a row matrix composed of $m 1$ 's. On using (i) of this theorem we get

$$
\left[s_{1}, s_{2}, \cdots, s_{r}\right] B=[c, c, \cdots, c]
$$

where $s_{i}$ denotes the sum of the $i$ th column of $A$. Transposing the relation we get

$$
B^{\prime}\left[\begin{array}{c}
s_{1} \\
s_{2} \\
\vdots \\
s_{r}
\end{array}\right]=\left[\begin{array}{c}
c \\
c \\
\vdots \\
c
\end{array}\right] .
$$

Received by the editors December 1.1953 
The right-hand side of (1) is a column matrix of $n c$ 's. Now rank $B^{\prime}$ $=$ rank $B=r$ and consequently $r$ of the $n$ rows of $B^{\prime}$ are linearly independent. Consider the corresponding $r$ equations involved in (1). As the coefficient matrix is nonsingular, these equations have a unique solution $s_{1}, s_{2}, \cdots, s_{r}$. Since the sum of the coefficients in any of the $r$ equations is $b$ by condition (ii) of this theorem, the unique solution is easily seen to be $s_{1}=s_{2}=\cdots=s_{r}=c / b$. Of course, $b$ cannot be the null element of the field-otherwise the rank of $B$ is less than $r$. This completes the proof.

If the column sums of $A$ are equal to $a$, then the equality of the column sums of any of $B$ and $C$ implies the equality of the column sums of the other and then $a b=c$-without any consideration of rank. This is easy to prove.

We now use Theorem 1 to establish a property of certain combinatorial arrangements. Suppose a "design" satisfies conditions (i) and (ii) of the first paragraph. List the $v$ varieties in a column and the $b$ blocks in a row. Construct an incidence matrix $A$ of the design by putting 1 or 0 in the $(i j)$ position of the matrix according as the $i$ th variety occurs in the $j$ th block or not. A design will be called nonsingular if $\operatorname{rank} A$ (or what is the same thing, $\operatorname{rank} A A^{\prime}$ ) is equal to the number of blocks.

Theorem 2. If, in a nonsingular design, all the varieties appear an equal number of times, and if the total number of objects in all the blocks containing any particular variety is a constant, then any two blocks contain the same number of objects.

Let each variety occur $r$ times in the design. Then the column sums of $A^{\prime}$, the transpose of the incidence matrix, are all $r$. If $A A^{\prime}=\left(\lambda_{i j}\right)$, $i, j=1,2, \cdots, v$, then $\lambda_{i i}=r, i=1,2, \cdots, v$, and $\lambda_{i j}$ is equal to the number of blocks in which $i$ th and $j$ th varieties occur together. Consider a fixed $i . \lambda_{i j}$ then can be taken as the number of times the $j$ th variety appears in the $r$ blocks containing the $i$ th variety. So $\sum_{j=1}^{0} \lambda_{i j}$, which is the column sum of the $i$ th column of $A A^{\prime}$, is equal to a constant, say $\lambda$, by our assumption. Consequently taking $B=A^{\prime}$ in Theorem I we get Theorem 2 .

A particular case of this theorem immediately extends Ryser's result to symmetrical group divisible designs - a type of designs which are being extensively studied at present $[3 ; 4]$.

\section{REFERENCES}

1. K. N. Majumdar, On some theorems in combinatories relating to incomplete block designs, Ann. Math. Statist. vol. 24 (1953) pp. 377-389. 
2. H. J. Ryser, A note on a combinatorial problem, Proc. Amer. Math. Soc. vol. 1 (1950) pp. 422-424.

3. R. C. Bose and W. S. Connor, Combinatorial properties of group divisible incomplete block designs, Ann. Math. Statist. vol. 23 (1952) pp. 367-383.

4. R. C. Bose, S. S. Shrikhande, and K. N. Bhattacharya, On the construction of group divisible incomplete block designs, Ann. Math. Statist. vol. 24 (1953) pp. 167-195.

Delhi University 